\title{
Management of Chronic Aortic Regurgitation. From Physician to Surgeon
}

\author{
Flávio Tarasoutchi, Max Grinberg
}

São Paulo, SP - Brazil

The emphasis on the importance of the natural history of aortic regurgitation, discussed by Spagnuolo et al ${ }^{1}$ in 1971 established the predictive criteria of clinical evolution and stimulated the development of reliable methods for follow-up of patients with aortic regurgitation.

Since the decade of the ' 80 s, the development of echocardiography has added imaging and other indices (for example, the so-called indices of ventricular function $)^{2-6}$ and perfected the tracing of the complex adaptive, ventricular remodeling.

It is known that an increase in ventricular cavity size with a final diastolic volume of $>150 \mathrm{~mL} / \mathrm{m}^{2}$ or systolic diameter $>70 \mathrm{~mm}$ and an index of end systolic volume $>60 \mathrm{~mL} / \mathrm{m}^{2}$ or systolic diameter $>55 \mathrm{~mm}$ is associated with the imminent onset of symptoms. But should these indices be the decisive parameters used by the physician in deciding whether to refer the patient to the surgeon?

Undoubtly, cardiac imaging data strengthen the decision about the best therapeutic approach for treating each phase of the natural history of aortic regurgitation, a complex ${ }^{7}$ physiopathologic valvular heart disease, by associating volume and pressure overload. However, the indices of cardiac imaging obtained are not sufficient and may lead to errors in evaluation if not interpreted from a clinical point of view, because no reliable index for determining prognosis exists. For this, we should remember that the complexity of interaction between pre and afterload causes difficulties in the interpretation of left ventricular function as the exact representation of myocardial function.

Values as high as $75 \mathrm{~mm}$ diastolic diameter and $55 \mathrm{~mm}$ systolic diameter were, in the early ' 80 s, true, exact, accurate indicators of a worse postoperative prognosis. Presently they are seen in a significant percentage of good evolution, even as normal in the late postoperative period, due to the present efficiency of intraoperative myocardial protection. Still in the decade of the ' 80 s, Carabello et al ${ }^{8}$ questioned the isolated use of the so-called worse prognostic indices in patients with severe aortic regurgitation and left ven-

Instituto do Coração do Hospital das Clínicas - FMUSP

Mailing Address: Flávio Tarasoutchi - InCor - Divisão Clínica - Av. Dr. Enéas C. Aguiar, 44 - 05403-000 - São Paulo, SP - Brazil - e-mail: tarasout@uol.com.br tricular dysfunction. Of 14 patients with left ventricular dysfunction, according to the various indices described in the literature, 12 experienced improvement in symptoms, reduction in diameters, and increase in ejection fraction-postoperatively which indicates that a systolic diameter $>60 \mathrm{~mm}$ correlated better with prognosis.

It seems useful to go further than resting on evaluation by cardiac imaging alone, and-whenever possible functional capacity and ventricular function should be evaluated through treadmill testing or isotonic exercises, coupled with radioisotopic ventriculography ${ }^{9,10}$. The total period of stress and the ejection fraction response are factors that give greater objectivity to the subjective determination of functional class obtained during anamnesis.

However, contrary to that which occurs with other heart diseases, the retuction in left ventricular ejection fraction during exercise does not seem to mean intrinsic myocardial dysfunction or accentuated loss of cardiac reserve. As initially reported by Borer et al ${ }^{11}$, ejection fraction during exercise tends to decrease with the natural evolution of aortic regurgitation without causing a change in functional class, and therefore, does not necessarily reflect actual reduction in cardiac reserve, being more useful for determining the intervals between patient re-evaluation.

It must be emphasized that besides the lack of direct correlation between a retuction in left ventricular ejection fraction during stress and myocardial dysfunction, the eventual abnormal response is associated with the probability of lack of symptoms for a 36-month period ${ }^{9,10}$. Radioisotopic ventriculography with stress/exercise has specific importance for patients in whorn maintenance or elevations of left ventricular ejection fraction during exercise in relation to the basal value should indicate good cardiac reserve and that the patient only needs a yearly re-evaluation as long as the clinical status remains stable. On the other hand, in asymptomatic patients who have mild left ventricular dysfunction at rest and during stress/exercise and good reserve of left ventricular ejection fraction during exercise, the clinical management should prevail, which requires twice a year clinical re-evaluations and cardiac imaging, including exercise testing. It is worth emphasizing that the average rate of occurrence of left ventricular dysfunction, although low, $1.3 \%$ per annum, justifies obtaining images during six month intervals, in spite of the fact that may not necessarily reflect left ventricular dysfunction. 
In the decade of the '90s, the experience with arterial vasodilators during the natural history of aortic regurgitation lead clinicians to wonder about the limits of clinical and surgical treatment and to try to determine whether vasodilators take on the true function of pharmacological scalpels.

And now at the start of a new decade, where do we stand regarding the value of the clinical and complementary examinations and the exact proportion required based on common sense versus personal experience and reports in the literature?

According to the idea of avoiding precocious interruption of natural evolution and, thus not anticipating the morbimortality inherent to valvar prosthesis, it has been noted that with the use of hydralazine asymptomatic patients with aortic regurgitation ${ }^{12}$ with cardiac dilatation experienced a reduction in the diastolic volume index of the left ventricle, leading to the conclusion that a pharmacological influence may have occurred via action on after-load during the natural history of aortic regurgitation with normal left ventricular function.

Subsequent utilization of nifedipine ${ }^{13}$ reduced the incidence of symptoms and left ventricular dysfunction, as well as the need for surgical treatment $(15 \pm 3 \% \times 34 \pm 6 \%$-p $<0.0$ $01)$ in relation to the use of digoxin.

Nevertheless, vasodilators must be used with care, as they may mask symptoms that alert clinicians to the clinical manifestation of a hemodynamically severe aortic regurgitation, which brings up the question of whether their prolonged use affects the reliability of symptoms as markers for surgical therapy?

Therefore, we must keep asymptomatic patients medicated with vasodilators under clinical observation and perform frequent assessment of ventricular function through cardiac imaging methods.

It should be emphasized that the known results of the slowing down of the natural evolution of aortic regurgitation occurs in the presence of normal ventricular function. When left ventricular dysfunction and other symptoms occur, the use of vasodilators is only preoperative therapy, but use of vasodilators is not a therapeutic test and will not postpone the need for surgical treatment.

Nowadays, a consensus exists as to which type of vasodilator to prescribed. The angiotensin-converting enzyme inhibitor is preferred. Banaszewski et al ${ }^{14}$ demonstrated captopril's better hemodynamic effect over nifedipine's in asymptomatic patients with aortic regurgitation, especially during exercise.

But when not using a vasodilator, what can be expected? Our study ${ }^{15}$ of 68 patients $(85 \%$ with a rheumatic disease etiology) in the absence of a vasodilator concluded that after 10 years $50 \%$ remained asymptomatic, and, of the remaining who underwent surgery as they developed symptoms, no deaths occurred due to left ventricular dysfunction.

It should be noted that in a metaanalysis of 7 clinical trials including 490 patients with severe aortic regurgitation and normal ejection fraction not on vasodilators, the occu- rence of symptoms or left ventricular dysfunction was only $4.3 \%$ /annum and sudden death was rare, $0.2 \%$ /annum in patients followed-up for 6.4 years ${ }^{16}$.

Similar to our study, Tornos et al ${ }^{17}$ observed in the evaluation of 108 patients followed-up for 10 years, that death occurred in asymptomatic patients with normal function, finding only six cases of asymptomatic left ventricular dysfunction. As already observed by Bonow et al ${ }^{18}$, the excellent evolution of patients with severe aortic regurgitation, as long as normal ventricular function was present, emphasizes the finding that the on-set of left ventricular dysfunction preceding symptoms is infrequent.

The symptoms of aortic regurgitation of rheumatic etiology, which usually occur in the $4^{\text {th }}$ or $5^{\text {th }}$ decade of life, mark the clinical moment ${ }^{9}$ in which natural history must be transformed into postoperative history, Similar to aortic stenosis, $30 \%$ of patients are estimated to evolve to death in the first year, $65 \%$ in the second, and $87 \%$ up to the seventh year. It is worth noting that the symptoms do no occur due to a decline in systolic function (ejection fraction $<60 \%$ ), but due to the great increase in after-load and filling pressure, without alteration in myocardial function.

Recently, Klodas et al ${ }^{19}$ reported that functional class II is an indicator for immediate surgery in patients with severe aortic regurgitation, justifying the surgery by the results of better prognosis in relation to the prognosis of patients in functional class III/IV. We must emphasize that the age group studied was older (average age 55 years) than the group usually studied in our population (average age 30 years). There is no doubt that age is a predictor of a worse postoperative prognosis ${ }^{12,20}$, but this factor must be carefully applied to each case, as well as must other markers of a poor prognosis, such as systolic diameter variation rate, ejection fraction, and duration of symptoms. The few asymptomatic patients, in spite of left ventricular dysfunction, develop some degree of symptoms within 2-3 years.

In specific situations, $5-10 \%$ of patients with isolated chronic aortic regurgitation who undergo surgical treatment have annular aortic ectasia. The most frequent cause is Marfan's syndrome. Gott et $\mathrm{al}^{21}$ have recommended elective surgery when the diameter of the root of the aorta reaches values greater than $6 \mathrm{~cm}$, due to the risk of dissection or rupture of the aorta; however; this management approach has caused controversy. Follow-up for 18 months with echocardiography showed the development of aortic dissection in 3 of 11 patients with annular aortic ectasia and diameter of the aortic root larger than $5 \mathrm{~cm}$. At the same time, four patients with similar values did not develop dissection.

In conclusion, we should bear in mind that the decision regarding the ideal moment for surgery should be avoided when the patient is oligosymptomatic, as long as no etiopathogeny primarily related to the aorta or left ventricular dysfunction exists. Furthermore, it is obvious that care in the preoperative preparation, in surgical techniques, including essential myocardial protection, and efficient immediate postoperative measures perfected during recent years, cannot be neglected. 
At the end of the decade of the ' 90 s, the therapeutic challenge persists. On the one hand, with vasodilators the indication for surgery is postponed, and on the other hand, precocious surgery for those still in functional class II with normal ventricular function may be considered. Therefore, the interest in research regarding which is the ideal thera- py persists; for each moment of the natural history of aortic regurgitation, new surgical techniques with prostheses arise as do reliable, noninvasive indices of intrinsic myocardial contractibility. In this manner, we will be contributing to the preservation of the quality of life and life expectancy of patients with chronic aortic regurgitation.

\section{References}

1. Spagnuolo M, Kloth H, Taranta A, Doyle E, Pasternak B. Natural history of rheumatic aortic regurgitation. Criteria predictive of death, congestive heart failure, and angina in young patients. Circulation 1971; 44: 368-80.

2. Henry WL, Bonow RO, Borer JS, Ware JH. Observations on the optimum time for operative intervention for aortic regurgitation. I. Evaluation of results of aortic valve replacement in symptomatic patients. Circulation 1980; 61: 471-83.

3. Henry WL, Bonow OR, Rosing D, Epstein SE. Observations on the optimum time for operative intervention for aortic regurgitation. II. Serial echocardiographic evaluation of asymptomatic patients. Circulation 1980; 61: 484-93.

4. Carabello BA, Usher BW, Hendrix GH. Predictors of outcome in patients for aortic valve replacement in patients with aortic regurgitation and left ventricular dysfunction: A change in the measuring stick. J Am Coll Cardiol 1997; 10: 991.

5. Forman R, Firth BG, Barnard MS. Prognostic significance of preoperative left ventricular ejection fraction and valve lesion in patients with aortic replacement. Am J Cardiol 1980; 45: 1120-5.

6. Gaasch WH, Carroll JD, Levine HJ, Criscitiello MG. Chronic aortic regurgitation: prognostic value of left ventricular end-systolic dimension and end- diastolic radius/thickness ratio. J Am Coll Cardiol 1983; 1: 775-82.

7. Wisenbaugh T, Spann JF, Carabello BA. Differences in myocardial performance and load between patients with similar amounts of chronic aortic versus crhonic mitral regurgitation. J Am Coll Cardiol 1984; 3: 916-23.

8. Carabello BA, Williams H, Gash AK. Hemodynamic predictors of outcome in patients undergoing valve replacement. Circulation 1986; 74: 1309-16.

9. Tarasoutchi F, Grinberg M, Parga F J , et al. Symptoms, left ventricular function, and the timing of value replacement surgery in patients with aortic regurgitation. Am Heart J 1999; 138: 477-85.

10. Tarasoutchi F, Grinberg $M, P \operatorname{Parga} F^{\circ} J$, et al. Relação entre função ventricular esquerda e desencadeamento de sintomas na insuficiência aórtica crônica severa. Arq Bras Cardiol 1995; 64: 301-9.

11. Borer JS, Bacharach SL, Green M, Kent KM, Henry WL. Exercise-induced left ventricular dysfunction in symptomatic and asymptomatic patients with aortic regurgitation:assessment with radionuclide cineangiography. Am J Cardiol 1978; 42: 352-7.

12. Zile MR. Chronic aortic and mitral regurgitation. Choosing the optimal time for surgical correction. Cardiol Clin 1991; 9: 239-53.

13. Scognamiglio R, Rahimtoola SH, Fasoli G, Nistri S, Volta SD. Nifedipine in asymptomatic patients with severe aortic regurgitation and normal left ventricular function. The New England J Med 1994; 331: 689-94.

14. Banaszewski M, Rydlewska Sadowska W, Rubkiewicz S. Captopril or nifedipine? Comparison of rest and exercise acute effects and long-term therapy in chronic isolated asymptomatic moderate to severe aortic regurgitation. J Heart Valve Dis 1998; 7: 488-99.

15. Tarasoutchi F, Grinberg M, Pomerantzeff $P$, et al. Should symptoms guide in patients with severe chronic aortic regurgitation? Eur Heart J 1998; 19: 632.

16. American College of Cardiology/American Heart Association. Guidelines for the management of patients with valvular heart disease. Circulation 1998; 98 : 1949-84.

17. Tornos MP, Olona M, Permanyer-Miralda G, Herrejon MP, Camprecios M. Clinical outcome of severe asymptomatic chronic aortic regurgitation: A long-term prospective follow-up study. Am Heart J 1995; 130: 333-9.

18. Bonow RO, Lakatos E, Maron BJ, Epstein SE. Serial long-term assessment of natural history of asymptomatic patients with chronic aortic regurgitation and normal left ventricular systolic function. Circulation 1991; 84: 1625-35.

19. Klodas E, Sarano-Eriquez M, Tajik J, Mullany CJ, Bailey KR, Seward JB. Surgery for aortic regurgitation in women. Optimizing timing of surgical correction in patients with severe aortic regurgitation: Role of symptoms. J Am Coll Cardiol 1997; 30: 746-52

20. Bonow RO, Nikas D, Eleftteriades J. Valve replacement for regurgitation lesions of the aortic or mitral valve in advanced left ventricular dysfunction. Cardiology Clinics 1995; 13: 73-83

21. Gott VL,Cameron DE, Pyeritz RE, et al.Composite graft repair of Marfan aneurysm of the ascending aorta: results in 150 patients. J Card Surg 1994; 9: 482-9. 